\title{
A New Voltage Reduction Scheme for HV Distribution Grid in The Spring Festival
}

\author{
Lianqing Zheng ${ }^{1}$, Xiaoxu Wang ${ }^{1}$, Lijun $\mathrm{Xu}^{2}$, Jiangtao $\mathrm{Xu}^{1}$ and Wenbin $\mathrm{Hao}^{3}$ \\ ${ }^{1}$ State Key Laboratory of Power Transmission Equipment \& System Security and \\ New Technology, Chongqing University, Chongqing 400044, China \\ ${ }^{2}$ Xinjiang institute of engineering, Xinjiang, 830000 \\ ${ }^{3}$ State Grid Sichuan Electric Power Research Institute, Chengdu 610016, China \\ 953362470@qq.com
}

\begin{abstract}
In order to lower the bus voltage of HV distribution network during holidays especially in the Spring Festival, a new voltage reduction scheme on account of electric coupling degree is proposed. The paper has proved the typical heterogeneous scale-free network features of power grid, when traditional voltage regulation measures are used up during the light load period, it's using electric coupling degree to identify the key substations owning the closest connections with other ones in the system and accordingly changes corresponding backup lines from hot-spare into cold standby mode to lower the voltage. Compared with load shedding depend on experiences of operating crew, the introduced scheme has obvious advantages of cutting less loads, which is more economic. Practical urban grid of west area of China indicates the proposed method has greatly optimized the voltage profile of the total network which is available and convenient and beams good application prospects.
\end{abstract}

Keywords: HV distribution Network Voltage reduction, Heterogeneous Network, Electric Coupling Degree, Backup Line Access Mode

\section{Introduction}

In the early stage of power gird construction, urban grid was equipped with inductive reactive compensators on the balance analysis of typical light load modes according to the "Power system voltage and reactive power technical guidance" by Chinese energy department. However, with the vigorous growth of power network, high tension cables have almost replaced all the overhead lines in main urban regions. On equal voltage class and length of lines, the reactive voltage class and length of lines, the reactive power produced by HV cables lines has an exceptional growth compared with that by overhead lines, as a result, the network originally equipped with inductive reactive compensation can't fully absorb the reactive power and thus causing voltages of different class to rise up, especially during the light load period [1]. For transmission network operating during the Spring Festival, the situation is much severe, generally voltages at this time rise to the highest level throughout the year. To guarantee the operation of the network, transformer taps are first adjusted to lower the voltage of $220 \mathrm{kV}$ by regulating to the top grade at the cost of $110 \mathrm{kV}$ buses exceeding the limit seriously, therefore, adjusting $110 \mathrm{kV}$ bus voltages to the rated value becomes the focus of this paper.

Nowadays, exploration in the field of voltage regulation puts particular emphasis on switching compensating capacitors to boost voltage amplitude while there is few special researches and special theory on regulation of over-voltage arose by reactive power surplus

Funded by National Natural Science Foundation of China (51577020) 
[2]. Literature [3] introduces practical voltage regulation measures applied in Guang Dong power grid from 3 aspects: switching on reactive compensating devices, transformer tap adjustment and generator leading phase operation and analyses of each measure's advantages and disadvantages as well as the applicable conditions are detailed made. Liu Yan-cheng proposed direct algorithm of safe correction and control of ultra-voltage in power system in view of least square method [4-5]; Based on sensitivity analysis, literature [6-7] adopts the principle in IEEE 5-bus and 26-bus system and obtains good results. Although the above references are based on different mathematics mechanism, the control objects and measures are the same: taking traditional voltage-regulating one. Mid\&Low-voltage distribution network reconfiguration proposed in literature [8-12] is based on artificial intelligence methods. Through adjusting the network configuration of network in certain algorithm, the reactive power flow will be changed, proper combinations could lead to global optimum. With the very difference from one-supply-multiple-backup connection of transmission, special electric position doesn't allow frequent structure changes of the urban HV distribution network. References [13-15] made simulation of $500 \mathrm{kV}$ transmission and suggested cutting long lines under the condition of $\mathrm{N}-1$ to minimize the capacitive charging of the long $\mathrm{AC}$ transmission lines. Whereas the proposed scheme applicable to transmission isn't suitable for the research objective of this paper due to the discrepancy in operation modes. In particular, when extensiveness $110 \mathrm{kV}$ buses conducting over the limit, taking the measures of cutting corresponding lines is unavailable, because that will be a heavy workload and easily cause the crash of system. Essentially, the method in references [13-15] draws from experience while ignoring the complex network properties and its integrity.

Different connection modes and parameters varies the electric power's static and dynamic characteristics; therefore, it makes sense to improve the power quality based on the net's nature. For this reason, the paper defines the electric coupling connection index which reflects the degree of coupling between any bus with others in the network, ultimately to identify the key substations. Adjusting the key substations to the rated value could obviously contribute to the net-wide improvement of voltage quality.

\section{Voltage Reduction Scheme}

\subsection{Heterogeneous Scale-Free Network Features}

Most networks in real-world aren't random, a minority of so called Hub nodes tend to have majority connections, while the rest has little, normally, they meet Zipf's law [16]. Some scholars made analysis of the relation between degree distribution and power-law distribution and proved the typical heterogeneous scale-free network features of power grid [17]. However, the existing ways of key stations' identification in power flow tracks is based on topology structure which isn't the entire determinant of the power's dynamic characteristics, besides that, adjacent buses in electric distance are more coupled than those closed in topology, therefore it might draw wrong conclusion to focus on the network topology while ignoring the electrical coupling effect among buses. In the N-bus network, referred to the definition of degree index in complex network, the electrical coupling degree of bus" $\mathrm{i}$ " is identified as :

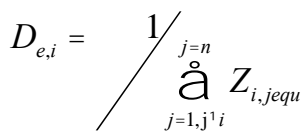

In expression $1, Z_{i, \text { jequ }}$ is the equivalent electric distance between bus $\mathrm{i}$ and $\mathrm{j}$, the importance of bus " $\mathrm{i}$ " posed in the network is expressed by $D_{e, i}$, which is used to identify key substations from electric perspective: the greater value, the stronger electrical coupling of bus "i" with the others. Hence, adjusting the corresponding bus to the rated value contrib- 
utes to improve other coupled over-limit bus voltage, which is possible to achieve global voltage improvement with the least changes of network connection.

\subsection{Mathematical Theory of Backup Lines Adjustment}

Study object of the paper: $110 \mathrm{kV}$ urban HV distribution network structures with closed loop design while running open loop, especially, $110 \mathrm{kV}$ substations connect with each other in the manner of "one-supply-one-backup". To accord the simplified model with practical system, we choose medium voltage side of $220 \mathrm{kV}$ transformer as the power supply of $110 \mathrm{kV}$ distribution network, $\mathrm{Sa}, \mathrm{Sb}, \mathrm{Sc}$ are constant power models of $110 \mathrm{kV}$ substations.

$\mathrm{Sa}$ and $\mathrm{Sb}$ is separately connected to side $\mathrm{A}$ and $\mathrm{B}$ and $\mathrm{Sc}$ forms "one-supply-one-back-up" connection mode with both sides, noted that the backup-line is set to heat standby the same as actual system, the simplified model is as follows:

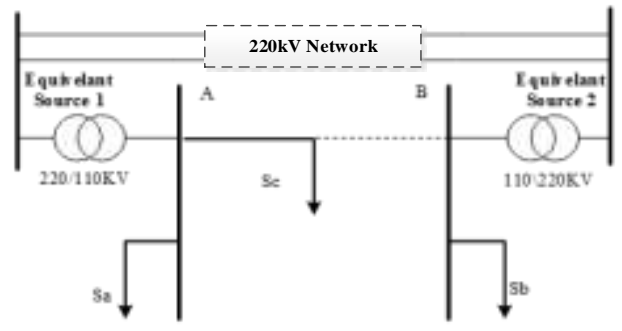

Figure 1. Simplified Model of 110kV urban HV Distribution Network

Making equivalent circuit of Figure 1:

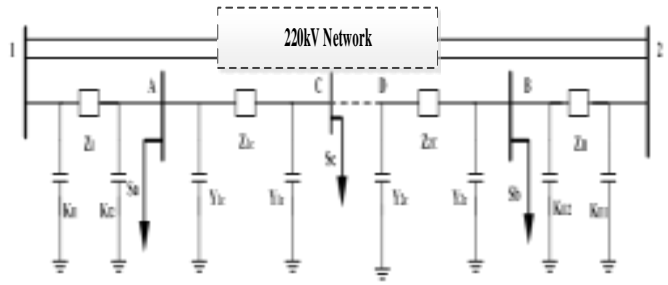

Figure 2. 110kV HV Distribution Network Equivalent Circuit

In Figure 2, although Sc isn't directly connected to the backup line, the reactive power of cable LBC still exists due to $U_{B}$ and the value equals that of switch on:

$$
Q_{c}=3 U I=\frac{U_{B}^{2}}{X_{c}}=\frac{U_{B}^{2}}{2 f_{c}}
$$

In equation $2, Q_{c}$ is the reactive power of cable line, $X_{c}$ is the capacitive reactance, $U_{B}$ is the bus voltage. Given end power: $\tilde{S}_{C}=P_{C}+j Q_{C}$ and given voltage of bus1 and bus2: $\dot{U}_{1}=\dot{U}_{2}=\dot{U}$, which stays unchanged because of the infinite capacity of generators. Assuming the per-unit voltage of every bus is $10^{\circ}$, calculating the power of bus 2 from side C step by step:

$$
\left\{\begin{array}{c}
\tilde{S}_{D B}{ }^{\prime}=\tilde{S}_{y D B}=Y_{D B}^{*} \\
\tilde{S}_{\mathrm{DB}}^{\prime \prime}=\tilde{S}_{D B}^{\prime}+\Delta \tilde{S}_{D B}=\tilde{S}_{D B}^{\prime}+S_{D B}^{\prime 2} Z_{D B} \\
\tilde{S}_{\mathrm{DB}}=\tilde{S}_{D B}^{\prime \prime}+\tilde{S}_{y D B}=\tilde{S}_{D B}^{\prime \prime}+Y_{B D}^{*}
\end{array}\right.
$$

Where $\tilde{S}_{\mathrm{DB}}^{\prime}, \tilde{S}_{\mathrm{DB}}^{\prime \prime} \tilde{S}_{\mathrm{DB}}, \tilde{S}_{y D B}, \Delta \tilde{S}_{D B}, Y_{D B}^{*}, Y_{B D}^{*}$ represents series branch end power, series branch top power, starting power, parallel branch power and parallel branch admittance. 
Similarly :

$$
\left\{\begin{array}{c}
\tilde{S}_{\mathrm{B} 2}=S_{B 2}^{\prime \prime}+\tilde{S}_{y 2 B} \\
S_{B 2}^{\prime \prime}=\tilde{S}_{B}+\tilde{S}_{D B}+Y_{B 2}^{*}+\left(\tilde{S}_{D B}+Y_{B 2}^{*}\right)^{2} Z_{B 2}
\end{array}\right.
$$

Where $S_{B 2}^{\prime \prime}, \tilde{S_{B 2}}$, represents series branch top power, starting power of LB2.

Resulted from $\dot{U}$ :

$$
\left\{\begin{array}{c}
\dot{U}_{B} \approx \dot{U}-\frac{S_{B 2}^{\prime \prime} \cos \varphi_{2} X_{2}}{\dot{U}}=\dot{U}-\frac{Q_{B 2}^{\prime \prime} X_{I}}{\dot{U}} \\
\dot{U}_{D} \approx \dot{U}_{B}-\frac{S_{D B}^{\prime \prime} \cos \varphi_{1} X_{2 D}}{\dot{U}_{B}^{*}}=\dot{U}_{B}-\frac{Q_{D B}^{\prime \prime} X_{2 C}}{\dot{U}_{B}^{*}}
\end{array}\right.
$$

Where $\cos { }_{1}, \cos { }_{2}$ is the head power factor of series branch of LDB and LB2. Disconnect the switch linked LBC locating besides port B and change heat standby into cold mode, gained that:

$$
\Delta \dot{U}_{B} \approx \frac{-Q_{C} X_{I}}{\dot{U}}
$$

\subsection{Flow Diagram of the Scheme}

As $110 \mathrm{kV}$ urban $\mathrm{HV}$ distribution network operates during holidays especially in the Spring Festival, the case of bus voltage running above the limit is notable and the reasons are detailed described in the introduction, when traditional measures can't meet the demand of voltage regulating, it's a good way to try changing the state of backup lines of key stations to get the reduction of charging flow, the concrete practices are described in the following diagram.

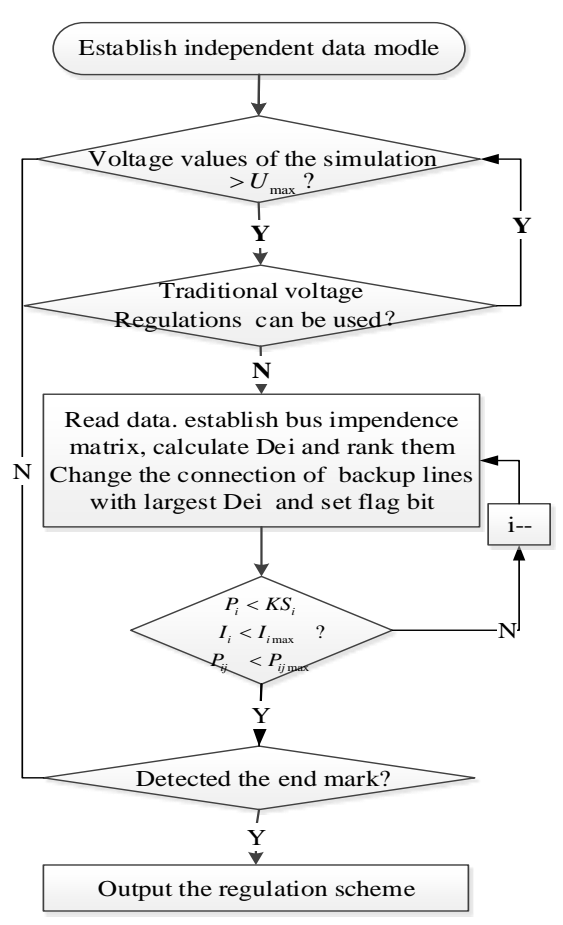

Figure 3. Flow Diagram of Voltage Adjustment based on Electric Connection

Step1: Establish independent grid data model during the Spring Festival and get convergent flow; 
Step2: Read voltage of $110 \mathrm{kV}$ substations and judge if there exist over-limit ones, if so, check whether traditional voltage measures could be used;

Step3: If the hypothesis is tenable, taking proper measures listed in the introduction and jump to step2 after regulation;

Step4: If not, building impedance matrix based on net's parameters and calculating the coupling connection degree of each node by formula 1 , select key substations whose $D_{e, i}$ is the biggest and read the connected backup-lines status. Then change the lines whose susceptance value is the largest from heat standby into cold mode while satisfying line thermal stability as well as interface flow:

$$
\text { st. }\left\{\begin{array}{c}
P_{i}<S_{i} \\
I_{i}<I_{i \max } \\
P_{i j}<P_{i j \max }
\end{array}\right.
$$

Remark the altered substation and keeps unchanged in the subsequent operations;

Step5: Recalculate the grid's power flow and repeat the above steps until detecting the end mark and jump out of the algorithm:

(1): the monitor bus voltages come to rated value;

(2): Consecutive alterations have little effect in regulation.

\section{Simulation Results}

The practical urban power grid of China consists of more than $40220 \mathrm{kV}$ substations and about $200110 \mathrm{kV}$ substations without any generator as well as phase modifier, obviously, it's typical of receiving network, the following is part of geographical wiring diagram.

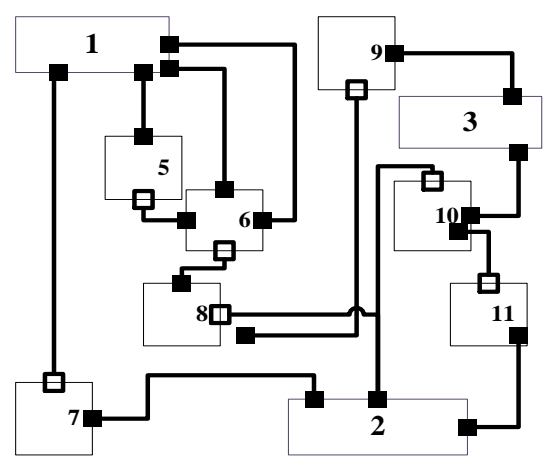

Figure 4. Simplified Models of Urban Grid in West Area of China

The rectangle blocks represent $220 \mathrm{kV}$ substations and the square ones represent $110 \mathrm{kV}$ substations, the wiring topology matches the practical power grid well.

After investigation, the charging reactive power is 900Mvar while the reactive power consumption of loads and equipped compensators amounts to only 800Mvar in the topology of the operation mode, due to the deficiency of inductive reactive compensators, as usual operation, transformer taps are adjusted to the top grade for the safety of power grid, as a result, $110 \mathrm{kV}$ buses operate over the limit seriously and the voltage distribution is as follows: 


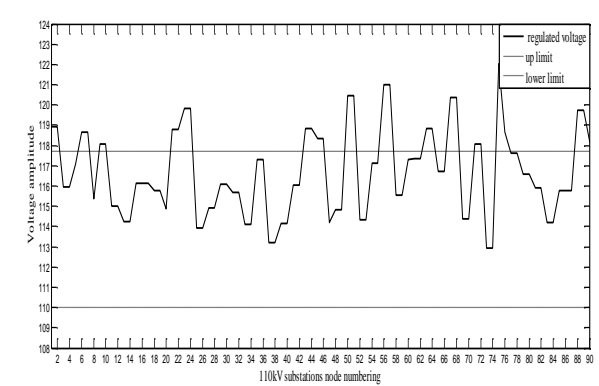

\section{Figure 5. Voltage Waveform of 110kV Substations after Traditional Voltage Adjustment during the Spring Festival}

Described in Figure 5, there are almost 39\% substations violating the limit, meanwhile, the dispersion degree is severe. According to the flow algorithm, it's important to establish independent data in accordance with actual operation mode during the Spring Festival, here selecting PSASP developed by Chinese electrical research institute as the simulation software. Getting the flow convergence, program on MATLAB to call the output files of PSASP and construct 576-bus impendence matrix. By formula, it's easy to obtain the rank of every node's coupling degree and identify the key substations. After changing 10 backup lines of 3 key substations from heat standby into cold mode, we get an ideal result and the typical parameters are listed:

Table 1. Voltage Before and After Network Optimization

\begin{tabular}{|c|c|c|}
\hline $\begin{array}{l}\text { Monitor } \\
\text { parameter }\end{array}$ & Before & After \\
\hline Bus 1 & $119 \mathrm{KV}$ & $117 \mathrm{KV}$ \\
\hline Bus 2 & $118.2 \mathrm{KV}$ & $116.5 \mathrm{KV}$ \\
\hline Bus 3 & $118.5 \mathrm{KV}$ & $117.3 \mathrm{KV}$ \\
\hline Bus 4 & $120 \mathrm{KV}$ & $117 \mathrm{KV}$ \\
\hline $\begin{array}{l}\text { Deviation } \\
\text { ratin }\end{array}$ & $35 \%$ & $6 \%$ \\
\hline Power factor & 0.992 & 0.935 \\
\hline
\end{tabular}

The result indicates the deviation ratio drops from $39 \%$ to $6 \%$ after regulations, besides that the power factor reduces from 0.98 to 0.95 , which equals to absorb 200Mvar reactive power through compensators. Comparison of voltage waveform before and after optimization is as follows: 


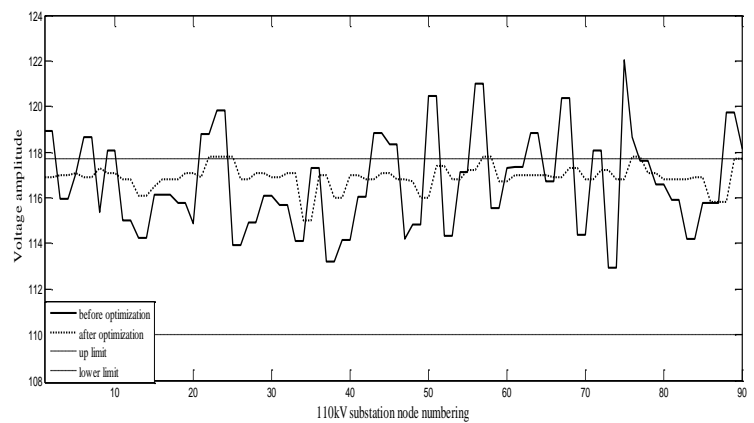

\section{Figure 6. Voltage waveform of $110 \mathrm{KV}$ substations before and after Optimization}

By contrast, the deviation degree gets reduced and the optimized curve is more smoothy.

\section{Conclusion}

According to the typical heterogeneous scale-free network features of power grid, the paper regards electric coupling index as the judgment of key substations, which covers the shortage of single consideration of connected relationships in topology, moreover, the proposed physical background suits actual grid better. Practical urban grid of Sichuan province indicates the method has effectively optimized the voltage profile of the entire grid and beams bright applying foreground in engineering, the further research of $110 \mathrm{kV}$ urban HV distribution network voltage regulation will probably be under this paper's guidance.

\section{References}

[1] R. Q. Che, B. Y. Liu and Z. Y. Zong, "Influence on cable lines to reactive power compension design calculation of $110 \mathrm{kV}$ substation”, Power Capacitor \& Reactive On Compensation, vol. 32, no. 1, (2012), pp. 13-15.

[2] H. L. Liu, "Research on optimal configuration of inductive reactive power compensators in regional power network", South china university of technology, (2012).

[3] P. Zhao, S. Cai, D. H. Dong and J. J. Sun, "Impact analysis of charging power of high-voltage cable on reactive power balance of power grid during light load periods", Zhejiang Electric Power, vol. 31, no. 2, (2013), pp. 20-23

[4] G. Y. Yang and L. Y. Li, "Analysis and countermeasures for Volt/Var control in Guangdong power grid during the period of the Spring festival", Power System Technology, vol. 31, no. 2, (2007), pp. 35-38.

[5] J. Wang, K. Q. Xu and M. Liao, "The operation experience of CSG during the spring festival, "Southern Power System Technology", vol. 7, no. 1, (2013), pp. 58-60.

[6] X. H. Ding, Y. Hong and L. Jun, "Simulation on the problem of high voltage in Hubei and Henan Provincial 500kV power system during 2008 spring festival", Power System Technology, vol. 32, no. 2, (2008), pp. 36-41.

[7] W. C. Meng and J. J. Qiu, "An artificial immune algorithm to distribution network reconfiguration", Proceedings of CSEE, vol. 26, no. 17, (2006), pp. 25-29.

[8] Q. Y. Chen, Y. Qian, W. R. Chen and C. H. Dai, "Multi-objective reactive power optimization and improvement of practical swarm algorithm", Power System Protection and Control, vol. 42, no. 5, (2014), pp. 129-135.

[9] H. C. Liu, X. H. Wang and J. M. Xiao, "Reactive power optimization based on group search optimizer", Power System Protection and Control, vol. 42, no. 1, (2014), pp. 93-99.

[10] H. D. Chiang and R. J. Jumeau, "Optimal network reconfigurations in distribution systems: part2 solution algorithms and numerical results", IEEE Trans on Power Delivery, vol. 5, no. 3, (1993), pp. 1568-1574.

[11] X. M. Li, Y.H. Huang and X. G. Yin, "A genetic algorithm based on improvement strategy for power distribution network", vol. 24, no. 2, (2004), pp. 49-54

[12] G. Y. Yang, Y. Li and L. Li, "Analysis and counter measures for Volt/Var control in Guangdong power grid during the period of Spring festival”, Power System Technology, Proceedings of the CSEE, vol. 31, no. 2, (2007), pp. 135-138.

[13] S. J. Li, R. J. Ren and S.Y. Zhou, "Fuzzy self-correction particle swarm optimization of environmental economic", Power system protection and control, vol. 42, no. 8, (2014), pp. 15-21. 
[14] Z. F. Liu, S.Y. Ge and Y. X. Yu, "A hybrid intelligent algorithm for loss minimum reconfiguration in distribution networks", Proceedings of CSEE, vol. 25, no. 15, (2005), pp. 73-78.

[15] X. M. Li, Y. H. Huang and X.G. Yin, "A genetic algorithm based on improvement strategy for power distribution network reconfiguration, "Proceedings of CSEE", vol. 24, no. 2, (2004), pp. 49-54.

[16] M. Zhou, J. H. Yang and H.B. Liu, "Internet topology modeling", Journal of Software, vol. 20, no. 1, (2009), pp. 109-123.

[17] Y. D. Tan and X. R. Li, "Critical Node Identification for Complex Power Grid Based on Electrical Distance", Proceedings of the CSEE, vol. 35, no. 1, (2014), pp. 91-98. 